\title{
COMUNIDADES DE FANS Y FICCIÓN TELEVISIVA. ESTUDIO DE CASO: EL MINISTERIO DEL TIEMPO (TVE)
}

\section{Communities of fans and television fiction. Case study: The ministry of time (TVG)}

Juan-Francisco Torregrosa-Carmona y Elena Rodríguez-Gómez
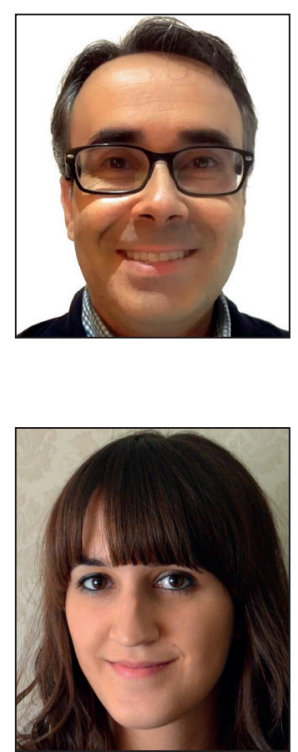

Juan-Francisco Torregrosa-Carmona es profesor titular del Departamento de Ciencias de la Comunicación y Sociología de la Universidad Rey Juan Carlos (URJC). Doctor por la Universidad Complutense de Madrid (2003), licenciado en Ciencias de la Información y en Derecho. Miembro de la Cátedra Unesco de Investigación en Comunicación y coordinador del Grado de Comunicación Audiovisual de la URJC. Perito judicial para cadenas públicas y privadas de televisión y autor de diversas publicaciones sobre información, comunicación y documentación. Conferenciante en Europa, América y Asia (Universidad de Pekín y Waseda University, de Tokio).

https://orcid.org/0000-0002-5983-5761

juanfrancisco.torregrosa@urjc.es

Elena Rodríguez-Gómez es graduada en Periodismo y Comunicación Audiovisual por la Universidad Rey Juan Carlos (URJC). Se ha especializado en ficción audiovisual, en concreto en aspectos relacionados con el campo del guion y las series televisivas. Se encuentra cursando el Máster de Guion de Cine y Televisión impartido por la Universidad Carlos III de Madrid (UC3M) en colaboración con el Sindicato de Guionistas ALMA.

https://orcid.org/0000-0002-8582-7052

rg.elena4@gmail.com

Universidad Rey Juan Carlos, Facultad de Ciencias de la Comunicación Camino del Molino, s/n. 28943 Fuenlabrada (Madrid), España

\section{Resumen}

Las comunidades de fans presentan una importancia creciente en las ficciones televisivas. Se analiza la serie El ministerio del tiempo (TVE) en relación con este fenómeno, cuya manifestación principal se produce en las redes sociales. Se han obtenido datos de una muestra de casi 3.600 personas autoidentificadas como "ministéricos". El análisis se realiza en el contexto de la convergencia de medios y la realidad transmediática de la televisión actual, en la que ejerce particular influencia la Red, hasta alcanzar a sus estrategias programáticas y a sus contenidos. El análisis se enfoca a la producción y promoción de creaciones audiovisuales dirigidas a una audiencia generalista. Esta investigación es de especial interés dentro de los fan studies porque constata la intensidad y la diversidad del caso estudiado y demuestra lo relevante que ha sido en la televisión nacional, especialmente en la pública, donde es difícil encontrar muestras de fandom con esta magnitud.

\section{Palabras clave}

Fans; Estudios de fandom; Ficción en televisión; El ministerio del tiempo; Producción audiovisual; TVE; Redes sociales; Twitter; Transmedia.

\begin{abstract}
Fan communities are increasingly important to television fiction series. The department of time television series (TVE) is examined in relation to this trend, with a focus on social networks. A sample of approximately 3,600 people calling themselves "ministerics" participated in the study. The analysis is carried out in the context of the convergence of media and transmedia, where the Net exerts particular influence on their program strategies and their contents, an it is focused to the production and promotion of audiovisual creations aimed at a general audience. This research is of special interest within the field of fan studies because it confirms the intensity and diversity of fans and shows the relevance that it has in national television, particularly in public television.
\end{abstract}

\section{Keywords}

Fans; Fandom studies; Television fiction; Scripted television; The department of time; Audiovisual Production; TVE; Social networks; Twitter; Transmedia. 
Torregrosa-Carmona, Juan-Francisco; Rodríguez-Gómez, Elena (2017). “Comunidades de fans y ficción televisiva. Estudio de caso: El ministerio del tiempo (TVE)". El profesional de la información, v. 26, n. 6, pp. 1139-1148.

https://doi.org/10.3145/epi.2017.nov.13

\section{Introducción, objetivos y metodología}

El ministerio del tiempo es una serie de ciencia ficción y fantasía de Televisión Española (TVE) creada por los hermanos Pablo y Javier Olivares y producida por Onza Partners y Cliffhanger. Se centra en las aventuras de un grupo de funcionarios españoles que deben viajar por el tiempo para evitar que la Historia sea cambiada. Empezó a emitirse el martes 24 de febrero de 2015; el resto de episodios se televisaron en prime-time los lunes. La primera temporada constaba de ocho capítulos. La segunda, transcurrió entre el lunes 15 de febrero y el lunes 23 de mayo de 2016, con 13 capítulos. La tercera temporada se estrenó el jueves 1 de junio de 2017.

El eje central de este artículo es el análisis de los ministéricos, autodenominación de los fans de El ministerio del tiempo. Se trata de determinar sus características, explicar qué aspectos de la serie son los que más gustan y conocer su interacción con las redes sociales. Se ha hecho un seguimiento de las mismas durante la segunda temporada (2016), así como un visionado completo de la serie, junto a la revisión documental de multitud de fuentes secundarias: monografías, artículos académicos y de prensa, blogs, productos fanmade... Todo con el objetivo de estudiar cómo son los seguidores de la serie, para así determinar los motivos que han hecho tan especial a este fenómeno fan.

\section{El ministerio del tiempo amplió su univer- so transmedia en la segunda temporada (2016) y presenta una base de fans a la altura de producciones internacionales}

La metodología de análisis es triangular y se han usado técnicas tanto cuantitativas como cualitativas. En la parte cuantitativa, un cuestionario ha proporcionado datos de una muestra de 3.599 personas seleccionadas de forma casual (durante el segundo semestre de 2016, hasta diciembre). Por su gran tamaño, es estadísticamente representativa y se pueden generalizar los resultados, aunque siempre existe un margen de error. La recolección cualitativa procede de un focus group en torno a los resultados de la encuesta, que fue organizado mediante el perfil Tiempo de fandom en Facebook, por tratarse de una red social que contaba con multitud de ministéricos registrados previamente y acostumbrados a debatir la serie en ella. En él se lanzaron 15 bloques de preguntas en un plazo comprendido entre el 7 de diciembre de 2016 y el 30 de enero de 2017, que contestaron una media de 10 personas. También se emplearon técnicas de observación participante en el Festival de Series 2016, en dos "quedadas ministéricas" (el 9 de abril de 2016 en Madrid y el 26 de noviembre de 2016 en Alcalá de Henares); en el preestreno de la segunda temporada, en la presentación del libro Dentro del ministerio del tiempo (24 de enero de 2016 en Fnac Callao, Madrid) y en redes sociales. Así se logró conocer de primera mano el comportamiento de los fans.

Los resultados permiten obtener unas conclusiones que constatan la relevancia y las características de la comunidad fan de esta producción.

\subsection{Justificación del objeto de estudio}

La serie ha sido uno de los grandes fenómenos de la televisión española en los últimos años, tal y como ha explicado Cascajosa-Virino (2015) y como se comprueba con esta investigación. Los ministéricos han conseguido con su presencia en redes sociales que se abra un debate sobre el valor de los audímetros tradicionales y su capacidad de medir el verdadero impacto de un programa (Establés-Heras; Rivera-Pinto, 2015). Tras la primera temporada, los fans promovieron la campaña \#TVErenuevaMdT para que hubiese más episodios (Establés-Heras; Rivera-Pinto, 2015) y recogieron 5.000 firmas en Change.org (Crisóstomo, 2016). También más de 5.000 personas pidieron su certificado oficial de ministérico (autogenerados a través de una web diseñada por el seguidor Manu Mateos). El estreno de la segunda temporada fue trending topic mundial y primero nacional en Twitter durante casi todo el día. Su amplia presencia en los medios ha cosechado gran éxito de críticas. Son numerosos los galardones: dos Premios Ondas a la Mejor Serie Española, premios ALMA 2015 y 2016 a mejor guion, Premios MIM y tres Premios Feroz. Debido al impacto que ha tenido en la ficción española es interesante estudiar su fandom.

\section{Comunidades de fans y ficción televisiva}

Es constatable que el medio hegemónico todavía hoy para consumir contenidos televisivos en los mercados europeos son los canales generalistas en abierto. Del mismo modo, una de las bases de sus programaciones sigue siendo la ficción (Delgado; Prado; Navarro, 2017).

La palabra fandom es un término que denomina al fenómeno fan. De origen inglés, surge de la contracción de "fanatic kingdom" ("reino fan") y se empleó por primera vez a principios del siglo XX para los aficionados al béisbol, aunque se popularizó en la ciencia ficción (Wilton, 2002). Ha evolucionado y su uso es extenso: puede aplicarse a la música o al deporte, por ejemplo. Cuando hablamos de seguidores de cine y series, lo hacemos del media fandom (Jenkins, 1992). Existen diferentes fandoms con nombre propio. Así, los seguidores de Doctor Who son denominados whovians, y los de Lost, losties. Multitud de comunidades online y materiales creados por fans están dedicados en exclusiva a series de televisión; algunas han sido renovadas por el apoyo de sus seguidores, como es el caso de Fringe y las películas basadas en las series Veronica Mars y Firefly (Guerrero-Pico, 2017).

Las comunidades crecieron de forma paralela a la ciencia ficción dominada por hombres a comienzos del siglo XX. 
Este género potencia especialmente al fan, según Cascajosa-Virino (2016, entrevista personal el 13 de noviembre). No fue hasta la década de los 60 y 70 cuando las mujeres empezaron a ocupar espacios para ellas y a reivindicarlos. Los fans consiguieron un nuevo protagonismo a partir de los años 90. Autores como Busquet-Duran (2012) los consideran pioneros en el uso de las tecnologías. Desde sus inicios y debido a que "fan" es una forma abreviada de la palabra latina "fanaticus" ("fanático"), el término ha presentado connotaciones negativas. Los prejuicios más comunes es que son consumidores descerebrados, inadaptados sociales, desexualizados debido a su íntimo compromiso con la cultura de masas, infantiles, incapaces de diferenciar realidad y ficción. Que su pasión es desmedida o poco seria. De ahí que en muchas ocasiones hayan sentido la necesidad de justificarse, alegando que si les atrae una serie es por cuestiones de alta cultura, como que los guiones los escriban figuras de renombre (Hills, 2002; Jenkins, 2002; Jenson, 1992; Fiske, 1992). También existe cierto recelo sobre el fandom porque rompe con la cultura dominante y se apropia de elementos de la cultura de masas (Jenkins, 1992) para darles nuevos significados.

Debido al interés social y en la industria que despertaron los media fandom (Hirsjärvi, 2013), surgieron en Estados Unidos los fandom studies. Henry Jenkins, una de las figuras más relevantes en este campo, afirma (1992) que el fandom aparece como respuesta a unas condiciones históricas específicas y, por tanto, permanece en cambio constante. Es mayoritariamente blanco y de clase media, además de femenino, pues el papel de las mujeres es clave en la historia del media fandom (Hellekson; Busse, 2006), especialmente como creadoras de contenido que adaptan los productos consumidos a sus intereses (Jenkins, 1992). Hoy en día es visto como un espacio igualmente femenino y de identificación queer (Stein, 2015, p. 22). De hecho, las series de temática LGBTI-como las recientemente populares Eyewitness y Skam- han generado comunidades muy grandes.

En la televisión actual, el fenómeno fan parece haber cobrado más fuerza gracias a internet. Se busca una audiencia activa que "observe con cuidado", para lograr fidelizar y retar a los espectadores (Espenson, 2010, p. 45), por lo que se empiezan a hacer distinciones entre "follower" (seguidor) y "fan" (Tulloch; Jenkins, 1995, p. 23). El primero sólo consumiría el producto, el segundo desarrollaría otras actividades en torno al mismo (Hills, 2002) y, además, estaría emocional e intelectualmente más involucrado. Ver una serie como fan implica niveles de atención diferentes de los que tiene un espectador más casual (Cox, 1990, citado por Jenkins, 1992). Además de una mayor valoración positiva del producto (Grandío, 2015) y una suerte de plenitud eudaimónica o felicidad especial. Algunas personas que se identifican así no tienen un compromiso directo con el fandom, para ellos ser fan es una forma de consumo, pero no necesariamente una clase de afiliación social (Jenkins, 2006).

\section{La influencia de las redes sociales ha} crecido: más de 50.000 tweets durante la emisión de algunos capítulos

La doctrina destaca que existen infinidad de espacios dedicados al universo fan. Se ha producido una mayor visibilidad de su cultura gracias a que "la Red proporciona un nuevo y poderoso canal de distribución" (Jenkins, 2008, p. 137). Han pasado de la marginalidad al mainstream en parte gracias a la visibilización de los millennial, esa generación de nacidos entre 1982 y 2004 . El colectivo ya es tenido en cuenta al generar valor económico, si bien este hecho no implica necesariamente un empoderamiento. Lo que sí se ha producido es un cambio de paradigma comunicativo, característico de la nueva convergencia tecnológica, económica y cultural: ahora los usuarios activos desempeñan un papel relevante. Y el estigma, aunque siga existiendo, es menor.

El fandom no demuestra que todas las audiencias sean activas, aunque sí prueba que no todas son pasivas (Jenkins, 1992). En 1996 se afianza el culto a las series de televisión y estallan fandoms como el de $X$-Files, que discutía la serie en la Web. El poder de las comunidades de fans se manifiesta, por ejemplo, cuando se organizan para lograr que sus series sean renovadas. Pero el fandom también tiene la capacidad de destruir; por ejemplo, eliminando personajes que no son de su interés -como fue el caso de Nikky y Paulo en Lost (Crisóstomo, 2016)- o intentando boicotear el producto que

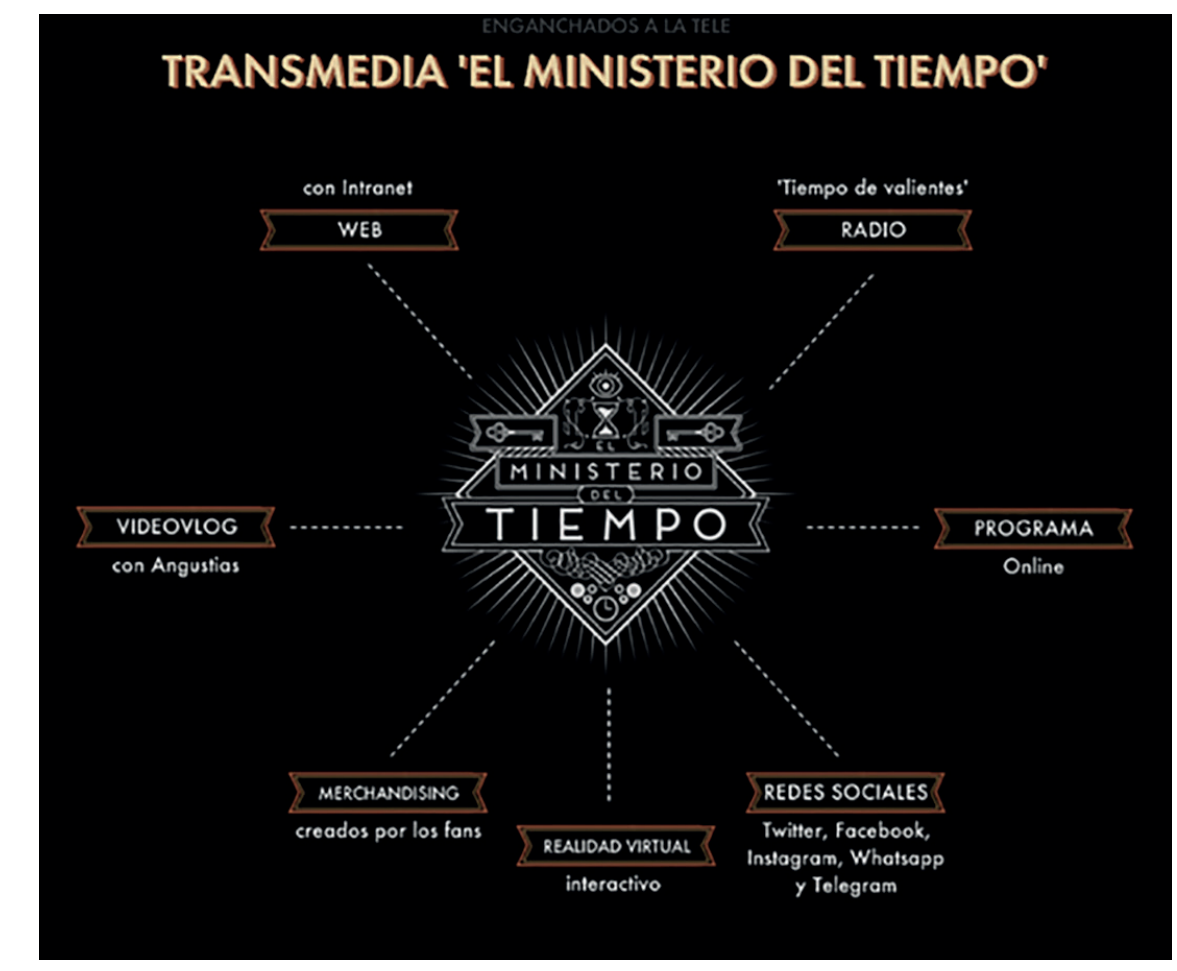


antes adoraban y a sus creadores, como en Los 100: tras la muerte de una de las protagonistas, muchos fans se indignaron en redes sociales y su showrunner Jason Rothenberg perdió al $12 \%$ de sus seguidores en Twitter. Esta enérgica reacción negativa, en parte se debe al fuerte vínculo que ha establecido el fan con el producto -pues a nivel neurobiológico la experiencia de consumir ficción es muy similar a la que se vive con situaciones reales (Paul, 2012)-, lo que le lleva a rechazarlo si siente que traiciona su manera de concebirlo.

En el siglo XX ya se evidencia la importancia de las narrativas transmedia -término acuñado por Jenkins en 2003 para hablar de la circulación de contenidos por canales diversos-, con ejemplos como Lost, que "nació como obra televisiva pero no tardó en expandirse a otros medios" (Scolari, 2011). Las series movilizan comunidades de inteligencia colectiva, como se ejemplifica en iniciativas como la Lostpedia (web tipo Wikipedia que recopila datos de la serie) o en que haya personas que subtitulen episodios de manera altruista y organizada (Zhang; Cassany, 2016). Lost también es un gran ejemplo de cómo los fans convierten el cariño que tenían a la serie en un odio igual de intenso, pues su polémico final dividió a los losties. Los espectadores actuales tienen hábitos nuevos gracias a la piratería y a las plataformas de vídeo bajo demanda (VOD) -que permiten el consumo personalizado de contenidos- así como a la emergencia de las segundas pantallas: ordenadores, tabletas o móviles (Cascajosa-Virino, 2016a). En este contexto, se populariza la figura del fan-creador o prosumidor frente a la de audiencia pasiva. El término prosumidor lo acuñó Toffler (1997) en su obra La tercera ola y hace referencia a personas que no se limitan a ser meros consumidores, sino que aportan otros bienes o servicios a la Red (Ramos-Serrano; Hernández-Santaolalla; Lozano-Delmar, 2012). De esta forma, la experiencia de ver televisión se transforma en una participación cultural más rica y compleja (Jenkins, 1992). ¿Y qué lleva a un fan a seleccionar un determinado producto para desarrollar este tipo de actividades? Según el propio Jenkins (1992), los eligen porque existe de antemano algún nivel de compatibilidad entre la construcción ideológica del texto y sus compromisos ideológicos; pero, en ocasiones, los productores no se sienten cómodos con las apropiaciones y reinterpretaciones de los fans e incluso surgen problemas por derechos de autor.

El fan se ha convertido en una pieza clave para promocionar la serie, aunque su nuevo rol también debe relativizarse, según Cascajosa-Virino (2016b). No quiere disfrutar en solitario del producto, sino que necesita compartir sus experiencias (Crisóstomo, 2016). Eso implica que la recepción audiovisual no sea exclusivamente individual e incluya actividades sociales en comunidad (Grandío, 2015), como demuestra que se organicen quedadas de fans o foros de debate online.

\section{Resultados}

El ministerio del tiempo se ha "europeizado en aspectos como la duración de capítulos, tratamiento visual cinematográfico y consideración hacia la autoría" (Cascajosa-Virino, 2016b, p. 165), logrando así una alta calidad en línea con los estándares que, según el antiguo ejecutivo de $H B O$ Collin
Callender, explican la mejora de las series norteamericanas. Como afirma Alberto Rey (2015),

"los ministéricos son fundamentales para entender la serie como ítem cultural y como fenómeno social".

Para determinar sus características principales, debemos preguntarnos qué lo ha convertido en un fenómeno cultural. La respuesta la tiene Scolari (2015), quien aporta elementos como "la gran intertextualidad", "guiños permanentes a la cultura mediática", "excelente realización audiovisual" y la "impecable interpretación de los actores". Pero, ¿̇es esto suficiente para generar un fenómeno fan tan grande? Según Grandío (entrevista personal el 14 de noviembre de 2016), la conexión entre la representación de emociones o conflictos humanos y las emociones del propio fan explica el germen del fandom.

\section{Los prosumidores se sitúan de forma mayoritaria entre 16 y 34 años}

Algunos ministéricos afirman haberse sentido identificados con personajes o situaciones; así, personas pertenecientes al colectivo LGBTI es más fácil que sientan afinidad con Irene Larra, como sucede con algunos Cayetaners. Por eso el $51,1 \%$ considera a los personajes un elemento clave de su interés por la serie. Sin embargo, es más amplio el número de personas que dan más importancia al factor histórico, pues el $73,2 \%$ ha votado esta opción. Además, hay bastantes seguidores apasionados de los géneros ciencia ficción y fantasía que han decidido verla debido a tal motivo. Por eso es fácil encontrar en redes sociales testimonios de personas que han afirmado que El ministerio del tiempo es la serie española que estaban esperando.

Entre los protagonistas preferidos, el gran ganador es Alonso de Entrerríos, el soldado de los tercios de Flandes interpretado por Nacho Fresneda, que acapara el 43,5\% de los votos: gusta por su sentido del honor, sus toques de humor y su evolución. Tras él aparece Amelia con un 27,6\% (más votada por mujeres, con el $30 \%$ de los votos, que por hombres, el $20 \%$ ) y Pacino, con un $8,7 \%$ (ligeramente preferido por mujeres: $8,7 \%$, frente a hombres, $8,4 \%)$. Después, Julián con el $7,2 \%$ (7,4\% hombres y un $7 \%$ mujeres). Esta implicación de los fans se consigue también tocando temas sociales que importan a su audiencia, como el feminismo, entendido en un sentido más práctico y social que académico o intelectual. En ese sentido, la jefa de la patrulla Amelia Folch se ha convertido en un icono y muchas personas (sobre todo mujeres) lo han destacado; como explica la ministérica $\mathrm{Ne}$ rea Pedraz, es posible que bastantes feministas se sientan "un poco Amelias del siglo XXI".

En cuanto al perfil de los seguidores, las personas que se autodenominan ministéricas son de todas las edades: entre los 10 y los 78 hay respuestas en el cuestionario, con una edad promedio de 32,3 y una mediana de 30,0 ; un rango muy amplio. Se debe a que funciona como una serie de nicho que también puede ser leída por público multitarget, aunque no capte todos los niveles de lectura (Pérez-Iglesias, 2015). Aunque el $52,6 \%$ son mujeres con educación supe- 
rior $(37,5 \%$ con estudios universitarios y un $15,1 \%$ con post-universitarios), también hay gente con formación básica o secundaria, además de estudiantes (31,3\%), desempleados $(13,4 \%)$, personas sin trabajo remunerado fuera del hogar (3,3\%) y jubilados (1\%), frente al $51,2 \%$ activos laboralmente.

Según Concepción Cascajosa (2016, entrevista), conviene tener en cuenta que las familias con hijos son una audiencia no cuantificada muy importante. Hay más seguidores en Madrid (21\%) y en Andalucía $(15,4 \%)$ que en otras regiones. La serie tiene bastantes fans latinoamericanos que suelen comentar en redes sociales. También existen subtítulos en otros idiomas y una pequeña comunidad fan de extranjeros, como demuestra que hayan respondido 177 personas de 42 países al cuestionario. Ha traspasado fronteras: se han vendido sus derechos a otros países, como China o Portugal, donde ya se emite Ministério do tempo (en RPT1).

Hay quien afirma que el éxito de la serie reside en haber atraído a "la generación Twitter, acostumbrada a consumir mucha televisión extranjera, y a verla como y cuando quiere" (Marcos, 2015, p. 200) y que además "ignoran, desprecian incluso, las series de televisión españolas" (Belmonte, 2015 , p. 55). En parte es cierto, muchos de los encuestados afirman pertenecer a fandoms extranjeros e internacionales, sobre todo a los de Doctor Who, Juego de tronos y Sherlock. Sin embargo, el 45,6\% también acostumbra a ver ficciones españolas, destacando aquellas de carácter histórico y otras tan populares como Mar de plástico, Víctor Ros, Olmos y Robles, Velvet, Cuéntame cómo pasó, El caso, Águila roja y La que se avecina. El 45,1\% afirma que El ministerio es su serie favorita; si analizamos este porcentaje vemos que, de ellos, han respondido afirmativamente el 35,3\% de los que nunca o casi nunca ven ficciones españolas, así como el $56,8 \%$ de los que sí suelen ver series españolas. Es decir, que son más proclives a considerar El ministerio su ficción favorita aquellos que no están acostumbrados a consumir productos televisivos extranjeros.

Las sorpresas y rupturas de expectativas y convenciones atraen a los espectadores (Mittel, 2007). El fandom televisivo actual está determinado por las nuevas formas de consumo, que despiertan un mayor deseo de participación (Ramos-Serrano; Hernández-Santaolalla; Lozano-Delmar, 2012). Así, encontramos a muchos seguidores que se han ido enganchando a lo largo de las temporadas (el $26,1 \%$ se sumó durante la emisión de la primera, mientras que un $7 \%$ lo hizo con la segunda). Resulta llamativo que, pese a ser una serie con multitud de ampliaciones transmedia, la gran mayoría de encuestados vean los episodios en directo en la televisión, especialmente los espectadores de mayor edad.

También en relación con el consumo de la serie, un $59 \%$ de personas vuelven a

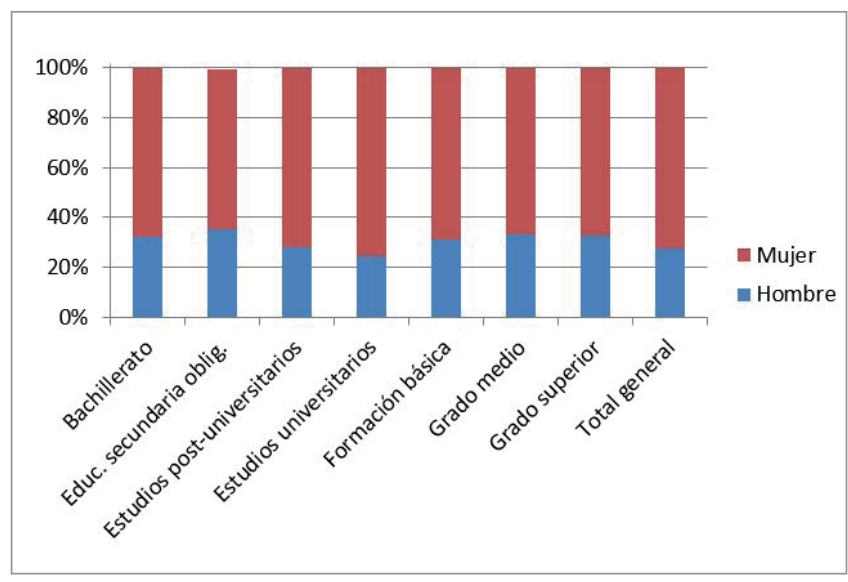

Gráfico 1. Nivel de estudios en función del género

ver los capítulos, de ellos sólo un 5,3\% la misma semana de su emisión, un $31,9 \%$ tiempo después y un $21,8 \%$ sólo si le ha gustado mucho; siendo las mujeres -sobre todo las jóvenes- las que más tienden al revisionado de episodios.

El $78,9 \%$ de los encuestados se considera ministérico y el $81,3 \%$ había oído el término antes. Este deseo de interacción hace que el $15,4 \%$ haya participado en eventos relacionados con la serie (el 8,3\% en foros y el 5,2\% en charlas). Como explica el fan Jorge González, así puedes hablar con personas con gustos similares y sentirte integrado en una comunidad a la que muchos consideran casi "una familia". Y es que, afirma la seguidora Marta Molins, se trata de un fandom muy unido. Sin embargo, Cascajosa-Virino (2016, entrevista) aclara que también hay muchas personas que se autodenominan ministéricas porque consideran que pertenecen a un grupo más amplio, pero que nunca van a participar en nada.

La narrativa transmedia se produce mediante dos vías: la oficial de los productores (contenidos top-down que circulan de arriba abajo, hacia los usuarios y reciben la denominación de "canon"; Scolari, 2011) y la de los fans (contenidos bottom-up creados desde el fondo de la cadena de producción; Guerrero-Pico, 2014). En ocasiones llegan a converger,

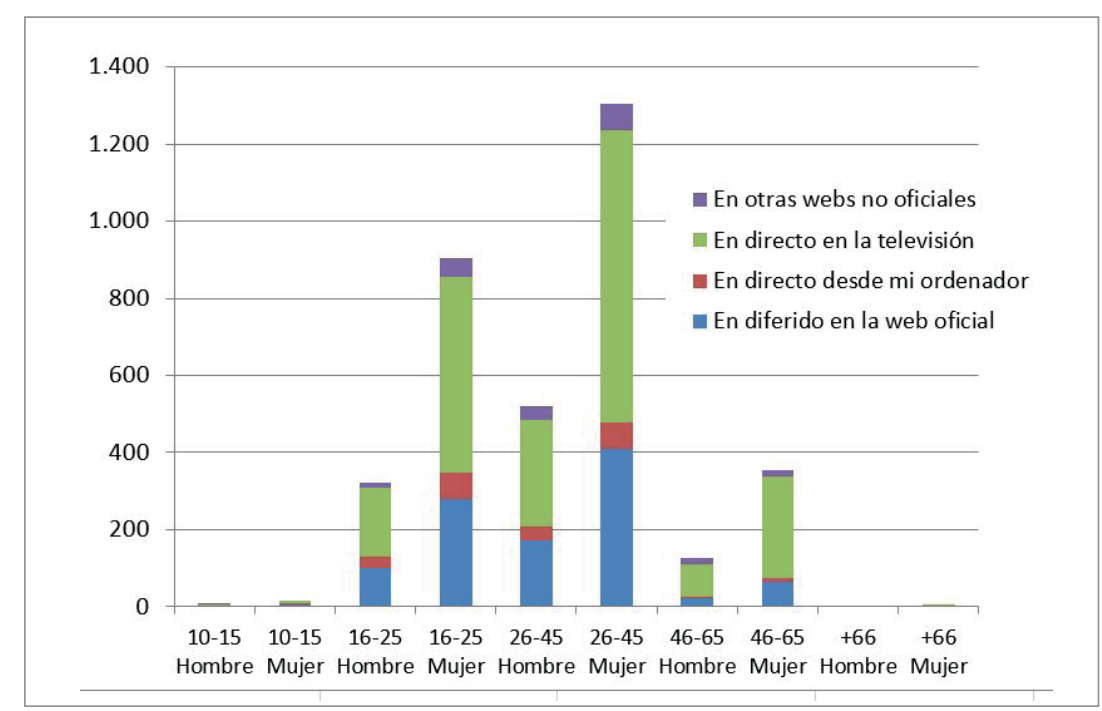

Gráfico 2. Consumo de la serie según rango de edad y género 


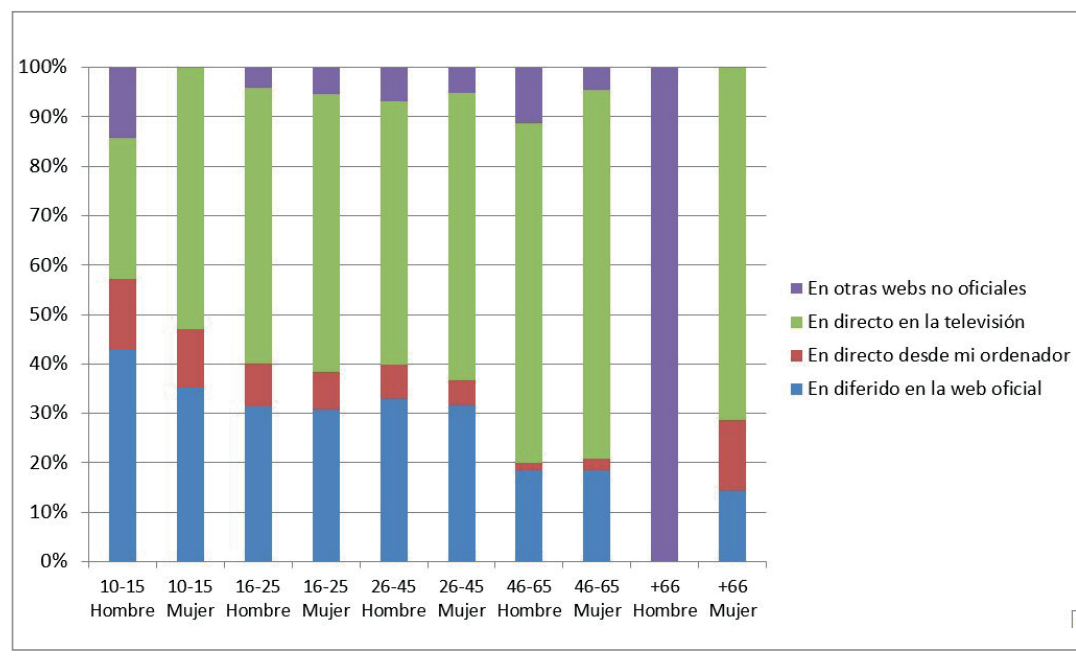

Gráfico 3. Consumo de la serie según rango de edad y género en porcentaje

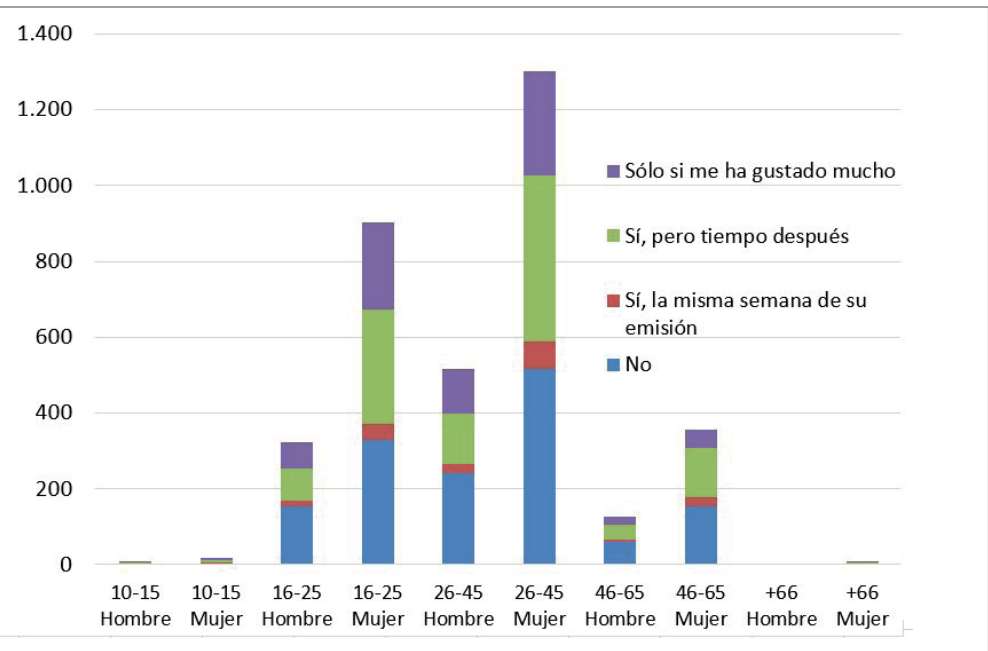

Gráfico 4. Revisionado de los episodios según rango de edad y género

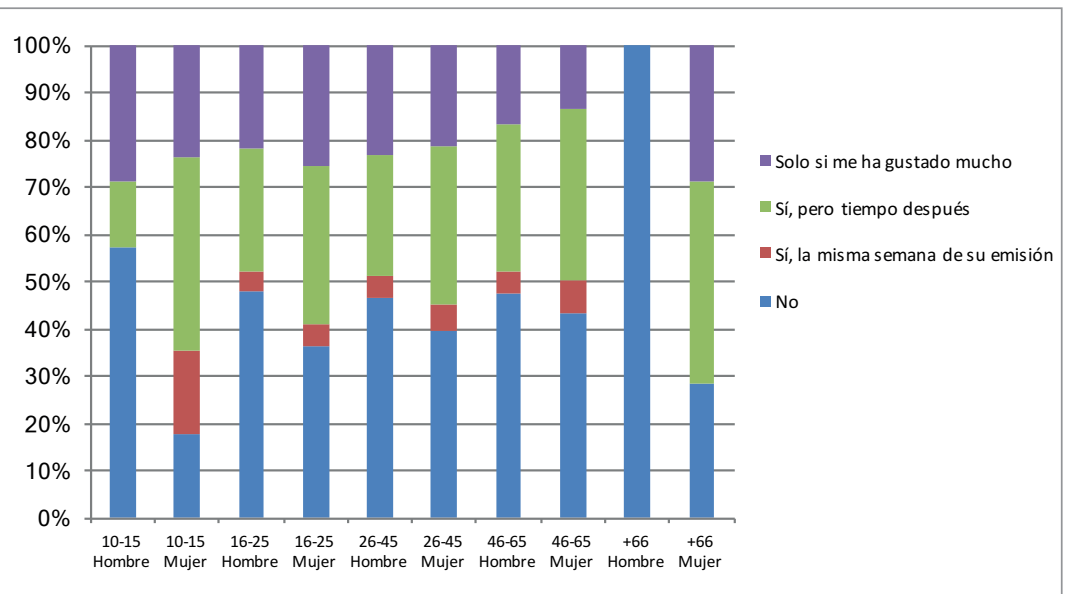

Gráfico 5. Revisionado de los episodios según rango de edad y género en porcentaje co medio (en este caso, la televisión) para luego expandirse a otros en función del éxito logrado (Scolari, 2016). Como explican sus responsables, el contenido transmedia oficial busca hacer que la creación siga viviendo para los fans incluso cuando no está en emisión. Es la serie con la que la cadena pública española ha innovado más fuera de la televisión, con muchos subproductos oficiales. Desde el inicio cuenta con web oficial, un foro de RTVE y los programas Los archivos del ministerio (un making of de media hora sobre la creación de cada capítulo) y La puerta del tiempo, emisión online en directo que debatía el episodio del día anterior. También existe la novela El tiempo es el que es (2016), escrita por Anaïs Schaaff y Javier Pascual. Con la segunda temporada aparecieron nuevas expansiones, que han aumentado en la tercera, con la llegada, entre otros, del cómic Tiempo al tiempo y un juego de mesa.

Para unificar el universo de seguidores, crearon la figura de Aurelio Pimentel, personaje ficticio y community manager que ofrece información privilegiada de lo que supuestamente está sucediendo dentro del Ministerio. Como híbrido entre ampliación fan y oficial encontramos el merchandising, diseñado por unos artistas que habían hecho fanarts previamente. Según los directivos, los fans no trabajan para ellos, sino junto a ellos, por eso eligieron a nueve para pedirles que diseñaran determinados productos. Sólo un 10,4\% de los encuestados ha comprado productos oficiales, aunque un $45 \%$ afirma que querría hacerlo. Las creaciones de los fans son tan relevantes como las oficiales. El $96 \%$ de los encuestados asegura no crear fanmades, siendo prosumidores sólo un $4 \%$, de los cuales el $30,7 \%$ produce fanarts y el $25,5 \%$ escribe fanfics, mientras que un $19,7 \%$ hace gifs. La mayoría de sus creaciones son subidas a Twitter (33,1\%), pero también emplean Tumblr $(19,2 \%)$, blogs (17,7\%) e Instagram (8,5\%). Expertos como Miranda-Galbe y Figuero-Espadas (2016) coinciden en que la serie presenta unas características que la hacen perfecta para desarrollar una estrategia transmedia que implique a sus fans, pues su arguaunque no es lo habitual. No ha sido este último el caso de $E I$ ministerio del tiempo: planificó su estrategia transmedia teniendo siempre en mente a los potenciales seguidores, convirtiendo la "unidireccionalidad tradicional de los textos" en bidireccionalidad (Maté-Puig, 2015), a diferencia de otras series, que empiezan como producto monomedia en un úni- mento presenta multitud de personajes, la posibilidad de que existan más patrullas de las que vemos en pantalla, viajes en el tiempo... Como dice Marcos Muñoz:

"El ministerio del tiempo propone un juego narrativo con unas reglas sencillas, pero tan lleno de posibilidades que resultaba tentador desde el principio". 


\subsection{Las redes sociales}

Con respecto a las cuentas oficiales en redes sociales, en octubre de 2016 tenían más de 36.600 seguidores en Twitter, 3.100 en Instagram, 35.700 "me gusta" en Facebook y centenares de mensajes en el foro de debate. En febrero de 2017 eran 106.000 seguidores en Twitter, 16.400 en Instagram, 91.732 "me gusta" en Facebook y 240 temas y 1.993 mensajes en el foro oficial. Un notable incremento. Gran parte del ruido mediático y de los éxitos logrados por los ministéricos han sido posibles gracias a su alta participación en redes sociales. La relación con las audiencias en general se ha acercado gracias al live tweeting, que produce un enriquecimiento de la experiencia de ver el contenido (Cascajosa-Virino, 2016a). Ese enriquecimiento se origina gracias a comentarios en directo a través de WhatsApp, Facebook y Twitter de los ministéricos que se divierten conversando y leyendo opiniones y reacciones. El $74,2 \%$ ha hablado de la serie en sus redes sociales; de ellos, el 55,4\% para comentar los capítulos y el $24,8 \%$ para interactuar con otros fans. Un $52 \%$ ha pedido la renovación de la serie mediante el hashtag \#TVErenuevaMdT.

Como Twitter es la red que más actividad registra como segunda pantalla y una de las que más compromiso genera entre los espectadores, los programas aprovechan su impacto para promocionarse, reuniendo todos los comentarios bajo una misma etiqueta o hashtag. En el caso analizado, las cuentas oficiales son las más citadas a la hora de comentar la serie y también las que acumulan más seguidores (Rodríguez-Mateos; Hernández-Pérez, 2015). Con cada episodio lograban colarse entre los trending topics el hashtag oficial e incluso otras palabras relacionadas, llegando a superar a programas muy populares. Así ocurrió con el episodio 9, el primero de la segunda temporada, cuya etiqueta \#VuelveMdT fue segundo trending topic a nivel internacional, por encima de la exitosa The walking dead, que ese mismo día regresaba con la segunda mitad de su sexta entrega. Durante la noche se publicaron más de 50.000 tweets sobre El ministerio del tiempo, cifra superior a los 45.000 comentarios en Twitter que se generaron con la emisión del último episodio de la primera temporada, por lo que el fenómeno fan online ha crecido.

\section{Una anomalía: multitud de fans de $E l$} Ministerio... nunca se habían involucrado en el famdom

En Facebook hay multitud de cuentas, siendo la más popular la oficial. Hay muchos perfiles (como clubs de fans italianos, argentinos y peruanos) y grupos, en su mayoría cerrados, aunque existen algunos públicos como "El ministerio del tiempo te necesita", con 726 miembros. Los más exitosos son "Funcionarios del ministerio del tiempo" (8.849 miembros, creado por Jaime Ángel el 25 de febrero de 2015) y “EI ministerio del tiempo" (8.882 miembros, iniciativa de Marga Adn Adorna el 15 de marzo de 2015).

Tumblr es una red social que ha tenido mucho peso entre el fandom, usada como comunidad de intercambio. El blog oficial:

http://ministeriodeltiempo.tumblr.com publica poco, sobre todo gifs e imágenes, además de repostear posts; menciona cuestiones como los subtítulos y hasta Aurelio Pimentel pide ayuda a los ministéricos para aprender a usarla. La cuenta oficial en Instagram (@elministeriodeltiempo) funciona como archivo confidencial del Ministerio. La serie cuenta con un canal de Telegram, con 1.600 miembros, y con una web oficial de gifs: http://www.rtve.es/television/20170322/ministerio-deltiempo-gifs/1505741.shtml

\section{La estrategia en redes sociales y una na-} rrativa transmedia fortalecen los universos de ficción televisiva

Las redes sociales son importantes y las estrategias en ellas -sumadas a la creación de una narrativa transmedia- fortalecen los universos de ficción televisivos. Sin embargo, según Cascajosa-Virino (2016, entrevista), también pueden generar un efecto túnel: existen ministéricos que no son activos online.

Finalmente, son varios los clubs de fans de los intérpretes. Aunque el $94,7 \%$ de los encuestados no forma parte de ninguno; un 2,1\% pertenece a Cayetaners (Cayetana Guillén Cuervo); un 1,5\% a Rodolferas (Rodolfo Sancho); un $1,4 \%$ a Fresnediers (Nacho Fresneda) y un 0,7\% a Clavij@s (Víctor Clavijo). Los fans aseguran que siempre se han mostrado encantados con la actitud de los actores y del resto del equipo de la serie hacia ellos y que el apoyo de los creadores ha logrado que sientan que forman parte de una comunidad.

\section{Conclusiones}

La comunidad de seguidores de El ministerio del tiempo es heterogénea: encontramos gente de todas las edades y condiciones, aunque en general triunfa entre personas con estudios superiores, especialmente mujeres.

Además, sus prosumidores tienen mayoritariamente entre 16 y 34 años y suponen un porcentaje pequeño sobre el total de fans, aunque significativo tanto por su peso como por los numerosos y variados contenidos que han creado.

La serie presenta unas características que han permitido el desarrollo de este fenómeno, como la posibilidad de expandir su mundo. El trasfondo histórico es lo que más gusta a los ministéricos, que son sobre todo personas interesadas en la cultura y la Historia.

El ruido generado en redes sociales ha sido fundamental para el reconocimiento y expansión de este fandom, pero también para la renovación de la serie en ambas ocasiones, a pesar de que esta última afirmación genera dudas a su showrunner.

Bastantes seguidores de El ministerio del tiempo estaban acostumbrados a pertenecer a otros fandoms televisivos $y$, por eso, se han convertido en ministéricos que han desarrollado actividades típicas de los mismos. Sin embargo, es interesante comprobar las anomalías que presenta este fenómeno fan, puesto que multitud de personas aseguran que nunca antes se habían involucrado en actividades relacionadas con el fandom. 
Queda claro que el apoyo de los creadores -en su mayoría a través de las redes sociales, tanto oficiales como privadas- ha sido un factor clave que ha unido, promovido y fomentado la aparición de fans implicados y, lo que es más importante, la existencia de ministéricos orgullosos de serlo. Afirman que se sienten integrados en una comunidad que consideran casi una familia.

Los ministéricos son la primera fanbase española que está prácticamente a la altura de las que existen para otras ficciones internacionales. De hecho, esta investigación resulta de especial interés dentro del ámbito de los fan studies porque constata la intensidad y la diversidad del fenómeno en el caso estudiado. Es revelador de la citada intensidad y variedad del movimiento fan de esta producción nacional el dato de que más de uno de cada tres (el 35,3\%) de los que nunca o casi nunca ven ficciones españolas consideren que El ministerio es su serie favorita; así como que el $78,9 \%$ de los encuestados se considere ministérico y que el $74,2 \%$ haya hablado de la ficción en sus redes sociales (un $24,8 \%$ para interactuar con otros fans).

La creación audiovisual analizada es un notable ejemplo de la primacía que conserva la televisión española generalista y en abierto, en línea con el contexto europeo, como vehículo para la atracción de consumos mayoritarios y con las producciones de ficción como una de las bases de su programación. En ese contexto, la ficción se ha confirmado hace años como un producto con mayor flexibilidad y capacidad de adaptación a las nuevas rutinas y costumbres vitales del espectador, de lo que los medios públicos televisivos han sido conscientes (García-de-Castro, 2008). Resultan contenidos a los que el fan vuelve, como demuestra que casi el $60 \%$ vean de nuevo los mismos capítulos. El perfil de quienes revisionan la serie a cada entrega es variado, según los resultados expuestos, aunque destacan las mujeres jóvenes (de entre 26 y 45 años), lo que confirma una vez más la relevancia del público femenino en las comunidades de fans.

\section{El trasfondo histórico de la serie es lo que más gusta a los ministéricos}

Este estudio demuestra lo relevante que ha sido y está siendo la serie El ministerio del tiempo dentro del conjunto de la televisión nacional, pero especialmente en la principal cadena pública ( $L a 1$ de TVE), donde es difícil encontrar muestras de fandom con esta magnitud.

La calidad de las series españolas ha suscitado debate tanto académico como, sobre todo, popular. Más allá de opiniones y valoraciones profesionales o estéticas, los estudios empíricos constatan que, a lo largo de las últimas décadas, las series de televisión se han convertido en uno de los contenidos estrella de las cadenas de televisión en España (Diego; Etayo; Pardo, 2011).

Los canales generalistas en abierto de titularidad pública no han sido ajenos a esta realidad de la industria audiovisual, compitiendo con las televisiones privadas por la atracción de una audiencia con amplio potencial. Es así hasta el punto de que al $60 \%$ de la población española le gusta este género. La mayoría de estos espectadores prefieren las series españolas frente a las norteamericanas. Dos factores podrían explicar tal preferencia: por un lado, la cercanía cultural de las historias narradas y de los personajes que las recrean; por otro, la gran habilidad de los canales comerciales para programar las series de producción propia en su franja más relevante, el prime time (Diego; Etayo; Pardo, 2011). Ambas condiciones se cumplen en el caso de El ministerio del tiempo, donde además destaca, como una tercera clave significativa, la cercanía e implicación de los creadores, que han sabido conectar con sus seguidores, logrando así que muchos ministéricos se sientan integrados en el fandom.

\section{Referencias}

Belmonte, Rosa (2015). "La coartada histórica y cultural”. En Cascajosa-Virino, Concepción (ed.). Dentro de El ministerio del tiempo. Alcalá de Henares: Léeme Libros. ISBN: 97884 15589310

Busquet-Duran, Jordi (2012). "El fenómeno de los fans e ídolos mediáticos: evolución conceptual y génesis histórica". Estudios de juventud, n. 96, Documentos 1. Jóvenes: Ídolos mediáticos y nuevos valores.

http://www.injuve.es/sites/default/files/2012/45/ publicaciones/Revista96_1.pdf

Cascajosa-Virino, Concepción (ed.). (2015). Dentro de El ministerio del tiempo. Alcalá de Henares: Léeme Libros. ISBN: 9788415589310

Cascajosa-Virino, Concepción (2016a). "Buscando al espectador serial desesperadamente: la nueva investigación de audiencias y la serie El ministerio del tiempo". Dígitos: revista de comunicación digital, n. 2, pp. 53-69. http://roderic.uv.es/handle/10550/53919

Cascajosa-Virino, Concepción (2016b). La cultura de las series. Barcelona: Laertes. ISBN: 9788475849942

Crisóstomo, Raquel (2016). “Fannibals ministéricos: el poder del Fandom". Index.comunicación: revista científica de comunicación aplicada, v. 6, n. 2.

http://journals.sfu.ca/indexcomunicacion/index.php/ indexcomunicacion/article/view/226

Delgado, Matilde; Prado, Emili; Navarro, Celina (2017). "Ficción televisiva en Europa (EU5): origen, circulación de productos y puesta en parrilla". El profesional de la información, v. 26, n. 1, pp. 132-140.

https://doi.org/10.3145/epi.2017.ene.14

Diego, Patricia; Etayo, Cristina; Pardo, Alejandro (2011). "La percepción sobre la calidad de las series televisivas en España: contraste entre el público y los profesionales". ZER. Revista de estudios de comunicación, n. 31, pp. 69-88. https://goo.gl/hqdBcV

Espenson, Jane (2010). "Playing hard to 'get'-how to write cult TV". En: Abbott, Stacey (ed.). The cult TV book. London-New York: I. B. Tauris, pp. 45-54. https://goo.gl/F8t6W8

Establés-Heras, María-Josefa; Rivera-Pinto, Diego (2015). "Ministéricos en Twitter y WhatsApp, o cómo el poder de los 
fans puede mover montañas". En: Cascajosa-Virino, Concepción (ed.), Dentro de El ministerio del tiempo. Alcalá de Henares: Léeme Libros, pp. 211-218. ISBN: 9788415589310

Fiske, John (1992). "The cultural economy of fandom". En: Lewis, Lisa A. (ed.). The adoring audience. Fan culture and popular media. London and New York: Routledge, pp. 30-49. http://paas.org.pl/wp-content/uploads/2014/07/Fiske.pdf

García-de-Castro, Mario (2008). "Los movimientos de renovación en las series televisivas españolas". Comunicar, v. 30, pp. 147-153. https://doi.org/10.3916/c30-2008-02-008

Grandío, María-del-Mar (2015). “El papel del fandom en la valoración positiva de una película: The World Hobbit Project y la audiencia mundial de El hobbit". Sphera pública, $\mathrm{n}$. 15 , v. 1 y 2 , pp. 21-33.

http://sphera.ucam.edu/index.php/sphera-01/article/ view $/ 253 / 233$

Guerrero-Pico, Mar (2014). "Webs televisivas y sus usuarios: un lugar para la narrativa transmedia. Los casos de Águila Roja y Juego de tronos en España". Comunicación y sociedad, n. 21, pp. 239-267.

http://www.scielo.org.mx/pdf/comso/n21/n21a10.pdf

Guerrero-Pico, Mar (2017). "\#Fringe, audiences, and fan labor: Twitter activism to save a TV show from cancellation". International journal of communication, v. 11, pp. 2071-2092. http://ijoc.org/index.php/ijoc/article/view/4020

Hills, Matt (2002). Fan cultures. London and New York: Routledge. ISBN: 9780415240253

Hirsjärvi, Irma (2013). "Alfabetización mediática, fandom y culturas participativas. Un desafío global". Anàlisi: quaderns de comunicació i cultura, n. 48, pp. 37-48.

https://doi.org/10.7238/a.v0iM.1964

Hellekson, Karen; Busse, Kristina (2006) (eds.). Fan fiction and fan communities in the age of the internet. New essays. Jefferson, North Carolina, USA: McFarland. ISBN: 978 0786426409

Jenkins, Henry (1992). Textual poachers. Televisión, fans \& participatory culture. London and New York: Routledge. ISBN: 9780415905725

Jenkins, Henry (2002). "Interactive audiences? The collective intelligence of media fans". The new media book, p. 157. https://labweb.education.wisc.edu/curric606/readings/ Jenkins2002.pdf

Jenkins, Henry (2006). "When fandom goes mainstream...". HenryJenkins.Org, 29 November.

http://henryjenkins.org/2006/11/when_fandom_goes_ mainstream.html

Jenkins, Henry (2008). Convergence culture: la cultura de la convergencia de los medios de comunicación. Barcelona: Paidós. ISBN: 9788449321535

Jenson, Joly (1992). "Fandom as pathology: The consequences of characterization". En: Lewis, Lisa A. (ed.). The adoring audience. Fan culture and popular media. London and New York: Routledge, pp. 9-29. ISBN: 0415078202 https://goo.gl/ZFuCdz
Marcos, Natalia (2015). “Una serie para la generación Twitter". En: Cascajosa-Virino, Concepción (ed.). Dentro de El ministerio del tiempo. Alcalá de Henares: Léeme Libros, pp. 199-202. ISBN: 9788415589310

Maté-Puig, Ignacio (2015). "Enseñando, que no es poco: El ministerio del tiempo en el aula de Historia". En: Cascajosa-Virino, Concepción (ed.). Dentro de El ministerio del tiempo. Alcalá de Henares: Léeme Libros, pp. 249-256. ISBN: 978 8415589310

Miranda-Galbe, Jorge; Figuero-Espadas, Javier (2016). “El rol del prosumidor en la expansión narrativa transmedia de las historias de ficción en televisión". Index comunicación. Revista científica de comunicación aplicada, v. 6, n. 2, pp. 115-134. http://journals.sfu.ca/indexcomunicacion/index.php/ indexcomunicacion/article/view/223

Mittel, Jason (2007). "Lost in a great story: Evaluation in narrative television (and television studies)". Just TV, 23 October.

https://justtv.wordpress.com/2007/10/23/lost-in-a-great-story

Paul, Annie M. (2012). "Your brain on fiction". The New York times, March 17.

http://www.nytimes.com/2012/03/18/opinion/sunday/theneuroscience-of-your-brain-on-fiction.html

Pérez-Iglesias, Jorge (2015): “'Chiquetete cantaría a Wagner con más solemnidad': referencialidad, cultura pop y el espectador ideal". En: Cascajosa-Virino, Concepción (ed.). Dentro de El ministerio del tiempo. Alcalá de Henares: Léeme Libros, pp. 233-240. ISBN: 9788415589310

Ramos-Serrano, Marina; Hernández-Santaolalla, Víctor; Lozano-Delmar, Javier (2012): “Fanadvertising y series de televisión". Revista comunicación, v. 10, n. 1, pp. 1211-1223. https://idus.us.es/xmlui/handle/11441/28867

Rey, Alberto (2015). "La puerta de los seriéfilos". En: Cascajosa-Virino, Concepción (ed.). Dentro de El ministerio del tiempo. Alcalá de Henares: Léeme Libros, pp. 3-6. ISBN: 978 8415589310

Rodríguez-Mateos, David; Hernández-Pérez, Tony (2015). "Televisión social en series de ficción y nuevos roles del documentalista audiovisual: el caso de El ministerio del tiempo". En: Index comunicación. Revista científica de comunicación aplicada, v. 5, n. 3, pp. 95-120.

http://journals.sfu.ca/indexcomunicacion/index.php/ indexcomunicacion/article/view/200/177

Schaaff, Anaïs; Pascual, Javier (2016). El tiempo es el que es. El ministerio del tiempo. Barcelona: Plaza \& Janés. ISBN: 9788401016974

Scolari, Carlos-Alberto (2011). "Lost. Narrativa transmedia, estrategias cross-media e hipertelevisión". En: Piscitelli, Alejandro; Scolari, Carlos-Alberto; Maguregui, Carina (eds.) Lostología. Instrucciones para entrar y salir de la isla. Buenos Aires: Editorial Cinema. https://goo.gl/cXexRU

Scolari, Carlos-Alberto (2015). "Regreso al ministerio del tiempo (I)". Hipermediaciones, 25 marzo. https://hipermediaciones.com/2015/03/25/ministerio_tiempo 
Scolari, Carlos-Alberto (2016). "Alfabetismo transmedia: estrategias de aprendizaje informal y competencias mediáticas en la nueva ecología de la comunicación". En: Telos: cuadernos de comunicación e innovación, n. 103. https://repositori.upf.edu/handle/10230/27788

Stein, Louisa (2015). Millennial fandom: Television audiences in the transmedia age. University Of lowa Press. ISBN: 9781609383565

Toffler, Alvin (1997). La tercera ola. Barcelona: Plaza \& Janes. ISBN: 9788401459306

Tulloch, John; Jenkins, Henry (1995). Science fiction audien- ces: Watching Doctor Who and Star Trek. London and New York: Routledge. ISBN: 9780415061414

Wilton, Dave (2002). "Word of the month: Fandom". Wordorigins.org, 1 July.

http://www.wordorigins.org/index.php/site/comments/ word_of_the_month_fandom

Zhang, Tian-Leticia; Cassany, Daniel (2016). “Fansubbing del español al chino: organización, roles y normas en la escritura colaborativa". BiD: textos universitaris de biblioteconomia i documentació, n. 37.

http://bid.ub.edu/es/37/tian.htm
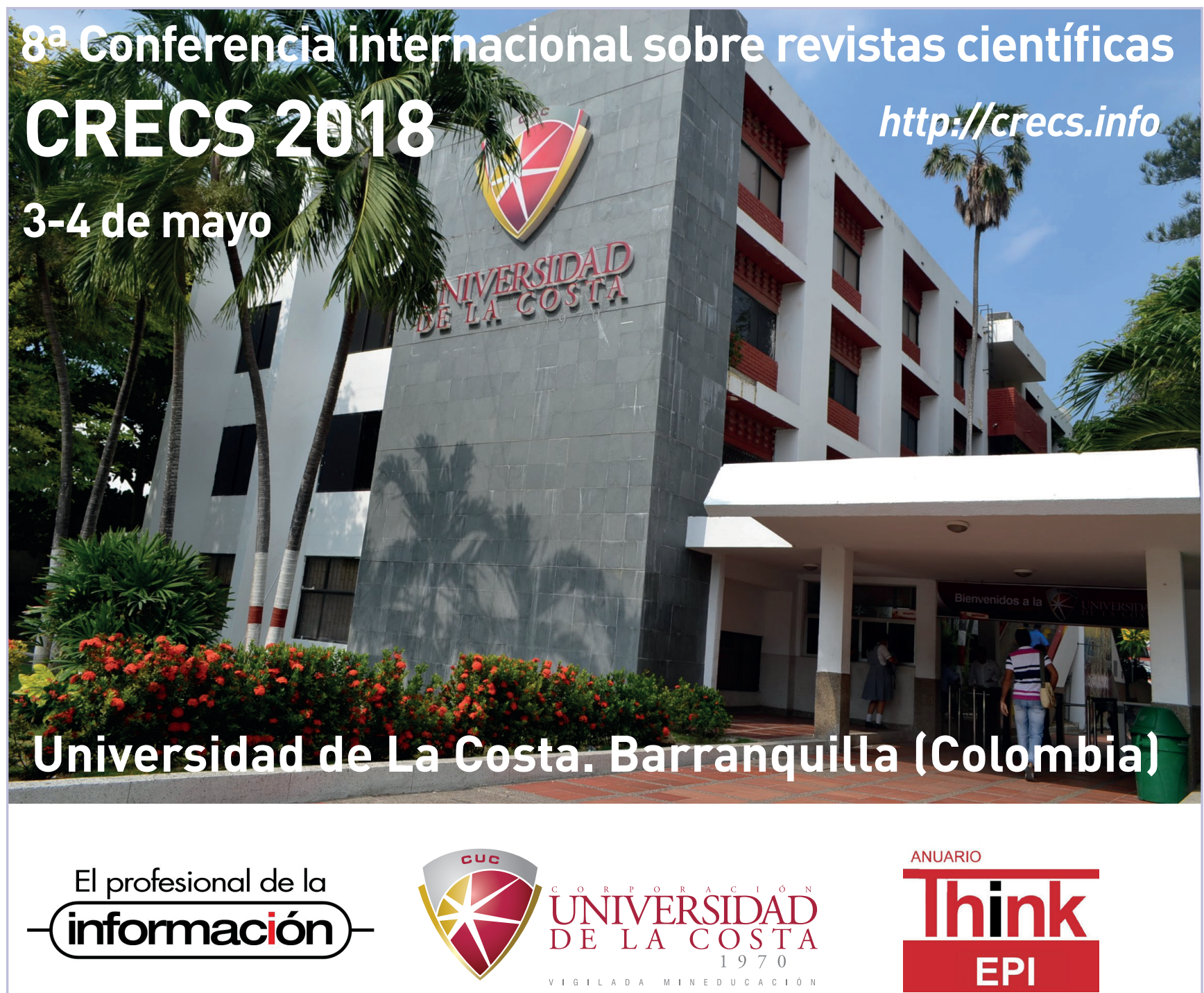

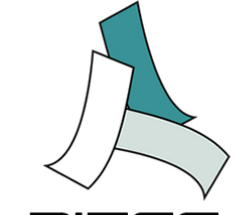

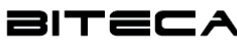
soluciones en infarmación

\section{Journals\&
Authors}
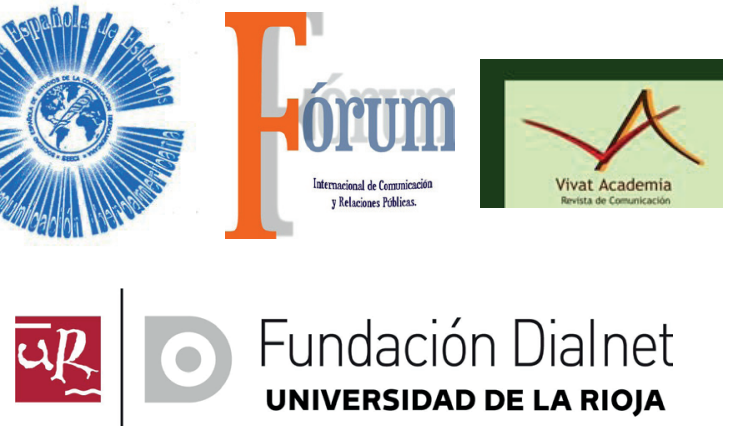

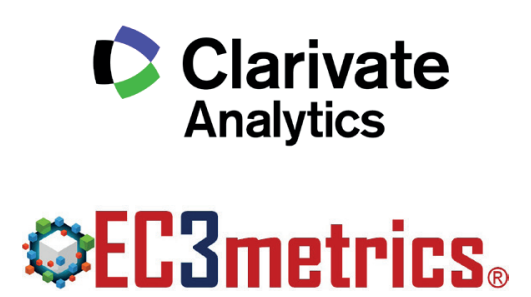

80 SCIMAGO 